\title{
Fobia social e habilidades sociais: uma revisão da literatura
}

\author{
Antonio Paulo Angélico \\ Universidade Federal de São Carlos \\ José Alexandre de Souza Crippa \\ Sonia Regina Loureiro \\ Faculdade de Medicina de Ribeirão Preto da Universidade de São Paulo
}

\begin{abstract}
RESUMO
Objetivou-se identificar nos indexadores Medline, PsycINFO, Lilacs e Scielo, no período de 2000 a 2005, estudos empíricos que abordem a fobia social e suas associações às habilidades sociais, visando a análise crítica das metodologias empregadas nesses estudos. Identificou-se 16 artigos, agrupados para análise em duas categorias: Modalidades Terapêuticas - Aplicação e Comparação de Intervenções Clínicas $(\mathrm{N}=6)$, e Caracterização do Repertório de Habilidades Sociais $(\mathrm{N}=10)$. Os estudos que compararam abordagens terapêuticas constataram a maior eficiência do treinamento em habilidades sociais (THS) para o desenvolvimento de repertório social mais competente, em comparação com a terapia cognitivo-comportamental. Os estudos de caracterização de perfis (10), relataram que os pacientes com fobia social apresentaram habilidades sociais mais deficitárias, apontando para o benefício potencial da participação em programas de THS. A análise crítica dos delineamentos apontou para a necessidade de novos estudos com amostras clínicas e não-clínicas, com alocação randômica dos participantes, com diagnóstico preciso e proposição de tarefas de interação contextualizadas, de modo a respaldar a generalização quanto à associação das habilidades sociais e fobia social, e evidenciar a funcionalidade e o processo pelo qual a ansiedade interfere no desempenho social.
\end{abstract}

Palavras-chave: fobia social; ansiedade social; habilidades sociais.

\begin{abstract}
Social phobia and social skills: a literature review

This study aimed to identify in the indexers Medline, PsycINFO, Lilacs and Scielo, in the period between 2000 and 2005, empirical studies that investigated social phobia in relation to social skills, followed by a critical analysis of the methodologies used by the studies listed. Sixteen papers were identified and grouped in two categories: Therapeutic Modalities - Application and Comparison of Clinical Interventions $(\mathrm{N}=6)$ and Characterization of the Social Skills Repertoire $(\mathrm{N}=10)$. The studies that compared therapeutic approaches evidenced the higher efficiency of the Social Skills Training (SST), for the development of more competent social repertoire, compared to cognitive-behavioral therapy. The studies of profile characterization (10) reported that the patients with social phobia presented a social skills deficit, suggesting the potential benefit of participation in social skills training programs. The critical analysis of the researches design pointed to the need of new studies with clinical and non-clinical samples, with random allocation of participants, precise diagnosis, and proposition of contextualized interaction tasks, in order to support the generalization of findings related to the association between the social skills and social phobia, and to demonstrate the functionality and process by which anxiety interferes with social performance.
\end{abstract}

Keywords: social phobia; social anxiety; social skills.

As habilidades sociais em geral são consideradas essenciais para os processos de ajustamento social dos indivíduos, portadores ou não de transtornos psiquiátricos (Angélico, 2004; Argyle, 1967/1994; Bandeira,
2003; Halford \& Hayes, 1995; Morrison \& Bellack, 1987; Turner, Beidel \& Townsley, 1992; Zigler \& Phillips, 1962). 
As habilidades sociais podem ser definidas como "diferentes classes de comportamentos sociais no repertório do indivíduo para lidar de maneira adequada com as demandas das situações interpessoais" (Del Prette \& Del Prette, 2001, p. 31). Tal conceito abrange o aspecto descritivo dos comportamentos verbais e não verbais apresentados pelo indivíduo diante das diferentes demandas das situações interpessoais. Uma demanda é entendida aqui como a ocasião diante da qual se espera um determinado desempenho social em relação a uma ou mais pessoas. No entanto, uma distinção entre esse conceito e o de desempenho social precisa ser feita. Desempenho social refere-se à emissão de um comportamento ou seqüência de comportamentos em uma situação social qualquer. Já o desempenho socialmente competente é aquele que, segundo Del Prette e Del Prette (2001), expressa uma leitura apropriada do ambiente social, que decodifica corretamente os desempenhos esperados, valorizados e efetivos para o indivíduo em sua relação com os demais, contribuindo na maximização de ganhos e na minimização de perdas para si e para aqueles com quem interage.

Considera-se que os déficits de habilidades sociais dificultam o funcionamento social do indivíduo e a sua capacidade adaptativa. Especificamente, questiona-se o envolvimento das habilidades sociais nos quadros clínicos de fobia social.

A fobia social tem sido considerada um grave problema de saúde mental pela sua alta prevalência e pelas incapacidades decorrentes no desempenho e nas interações sociais. Conforme os critérios diagnósticos do DSM-IV (American Psychiatric Association APA, 1994), os indivíduos com fobia social manifestam um medo excessivo, persistente e irracional de serem vistos comportando-se de um modo humilhante ou embaraçoso - pela demonstração de ansiedade ou de desempenho inadequado - e de conseqüente desaprovação ou rejeição por parte dos outros.

Conforme revisão empreendida por Furmark (2000), a investigação sobre fobia social e habilidades sociais tem sido inconsistente, considerando que embora os fóbicos sociais pareçam ter desempenhos inadequados como apontado em alguns estudos, isso poderia refletir inibição durante estados de alta ansiedade ao contrário de falta real de habilidades. Neste sentido, seria possível e desejável desenvolver ou estimular habilidades de enfrentamento no repertório destes indivíduos.
Ao se avaliar os tipos de déficits que um indivíduo pode apresentar em seu repertório de habilidades sociais, pode-se destacar: a) déficit de aquisição (caracterizado pela não-ocorrência da habilidade social diante das demandas do ambiente); b) déficit de desempenho (caracterizado pela ocorrência de uma habilidade específica com frequiência inferior à esperada diante das demandas do ambiente); e c) déficit de fluência (demonstrado pela ocorrência da habilidade com proficiência inferior à esperada diante das demandas sociais).

Considerando o papel da ansiedade na inibição do desempenho socialmente competente e a inconsistência de dados quanto à associação entre fobia social e habilidades sociais, buscou-se identificar na literatura indexada, no período de 2000 a 2005, artigos empíricos que abordem tal temática, visando a análise crítica das metodologias de pesquisa empregadas nesses estudos.

\section{PROCEDIMENTO}

Procedeu-se à identificação dos estudos empíricos relativos à temática "Fobia Social e Habilidades Sociais" com base em uma busca sistemática na literatura, usando os indexadores eletrônicos Medline, PsycINFO, Lilacs e Scielo, por meio do cruzamento das palavras-chave: social phobia, social anxiety, social skills e social skills training.

Incluíram-se estudos com participantes de ambos os sexos, adultos, procedentes de amostras clínicas, universitárias e da comunidade. Adotaram-se como critérios de exclusão estudos relativos a: (a) outras faixas etárias (crianças, adolescentes e idosos); (b) outros diagnósticos (outros transtornos e síndromes); (c) outras abordagens aplicadas à temática, como estudos farmacológicos ou exclusivamente psicométricos; (d) relatos de programas sobre modalidades de intervenções; (e) questões de pesquisa abordando a associação de ansiedade social e/ou habilidades sociais a desfiguração da face, problemas endócrinos, lesões cerebrais, implante coclear, enurese e adversidades sociais; e (f) associação de ansiedade social e/ou habilidades sociais às variáveis como auto-imagem, locus de controle, comportamentos internalizantes/externalizantes, stress e mecanismos de defesa.

Com base no procedimento de busca e aplicação dos critérios de inclusão e exclusão, foi identificado e analisado um conjunto de 16 artigos. 


\section{RESULTADOS}

Para a análise, os 16 artigos incluídos foram agrupados em duas categorias distintas: Caracterização do Repertório de Habilidades Sociais $(\mathrm{N}=10)$, e Modalidades Terapêuticas - Aplicação e Comparação de Intervenções Clínicas $(\mathrm{N}=6)$.

\section{Caracterização do repertório de habilidades sociais}

Quanto aos métodos de pesquisa empregados nos estudos incluídos nesta categoria, observou-se uma predominância de estudos de corte transversal (Alden \& Mellings, 2004; Baker \& Edelmann, 2002; Bögels,
Rijsemus \& De Jong, 2002; Christensen, Stein \& Means-Christensen, 2003; Horley, Williams, Gonsalvez \& Gordon, 2003; Sheffer, Penn \& Cassisi, 2001; Stopa \& Clark, 2000; Thompson \& Rapee, 2002; Wenzel, Graff-Dolezal, Macho \& Brendle, 2005) e apenas um estudo longitudinal (Strahan, 2003).

Estes estudos tiveram em comum as descrições de perfis de habilidades sociais apresentadas por indivíduos com fobia social generalizada ou com níveis altos de ansiedade social em medidas de auto-relato. Uma caracterização de aspectos específicos acerca dos delineamentos adotados nos estudos de descrição de perfis é apresentada na Tabela 1.

Tabela 1: Caracterização dos delineamentos adotados nos estudos de descrição de perfis

\begin{tabular}{|c|c|c|c|c|c|c|c|c|c|c|}
\hline \multirow{2}{*}{ DELINEAMENTOS } & \multicolumn{10}{|c|}{ ESTUDOS } \\
\hline & 1 & 2 & 3 & 4 & 5 & 6 & 7 & 8 & 9 & 10 \\
\hline № de participantes: - com fobia social generalizada & 18 & - & - & - & - & 25 & - & 15 & 20 & - \\
\hline - com outros transtornos de ansiedade & 18 & - & - & - & - & - & - & - & 20 & - \\
\hline - ansiosos socialmente & - & 36 & 26 & - & 13 & - & 62 & - & - & 55 \\
\hline - não-clínicos & 18 & 36 & 24 & 29 & 14 & 26 & 62 & 15 & 20 & 198 \\
\hline Indicadores de HS: - desempenho social & + & + & + & + & + & - & - & - & - & - \\
\hline - percepções interpessoais & - & - & - & - & - & + & + & + & + & - \\
\hline - desempenho acadêmico & - & - & - & - & - & - & - & - & - & + \\
\hline - habilidades de comunicação & - & - & - & - & + & - & - & - & - & - \\
\hline - competência social & + & - & - & + & + & - & - & - & - & + \\
\hline Contexto de coleta: a) tarefas de interação social & + & + & + & + & + & + & + & - & - & - \\
\hline - uso de dicas verbais pelo aux. pesq. & + & - & + & + & - & + & - & - & - & - \\
\hline - duração da interação social (em min.) & 9 & 5 & 10 & 6 & 30 & 5 & 15 & - & - & - \\
\hline b) apresentação de fotos com faces & - & - & - & - & - & - & - & + & - & - \\
\hline c) preenchimento de question. e escalas & + & + & + & + & + & + & + & + & + & + \\
\hline d) emprego de medidas fisiológicas & - & + & - & + & - & - & - & - & - & - \\
\hline Interlocutor na interação social: - auxiliar de pesquisa & + & + & + & + & - & + & - & - & - & + \\
\hline - parceiro afetivo & - & - & - & - & + & - & - & - & - & - \\
\hline - outro participante & - & - & - & - & - & - & + & - & - & - \\
\hline
\end{tabular}

As amostras dos estudos variaram de 27 a 253 participantes (mediana - 52), sendo constituídas por pacientes com diagnóstico de fobia social generalizada ou outros transtornos de ansiedade, indivíduos ansiosos socialmente e não-clínicos, de ambos os sexos, com idades variando entre 18 a 64 anos. A origem dos participantes foi predominantemente do ambiente universitário, seguido do ambiente clínico e da comunidade. Três estudos apresentaram critérios de exclusão para a composição da amostra, tendo em comum entre eles a condição dos pacientes satisfazerem os critérios diagnósticos para transtornos psicóticos, transtorno mental orgânico e adição a substâncias
(Alden \& Mellings, 2004; Baker \& Edelmann, 2002; Horley e cols., 2003).

Os objetivos dos estudos analisados focalizaram o papel da ansiedade sobre diversos indicadores de habilidades sociais. Tais estudos sugeriram que níveis altos de ansiedade social afetam negativamente: (a) o desempenho social, definido como desempenho comportamental molecular e molar exibido durante a interação social; (b) o desempenho acadêmico, avaliado pela taxa de abandono e aproveitamento acadêmico; (c) as habilidades de comunicação, definidas pelo conteúdo verbal e função da fala exibidos na interação com o parceiro afetivo; (d) as percepções interpessoais, 
definidas como julgamentos sociais, autopercepções, meta-percepções e percepção do outro, interpretação de eventos sociais e processamento de expressões faciais; e (e) a competência social, definida como o nível de proficiência com que as classes de comportamentos verbais e não verbais de um indivíduo são articuladas em um desempenho social bem sucedido.

Entre os diversos instrumentos e medidas empregados nos estudos, o Beck Depression Inventory (BDI) foi o mais utilizado (Alden \& Mellings, 2004; Baker \& Edelmann, 2002; Christensen e cols., 2003; Horley e cols., 2003; Thompson \& Rapee, 2002), seguido do Fear Negative Evaluation Scale (FNE) (Horley e cols., 2003; Thompson \& Rapee, 2002; Stopa \& Clark, 2000; Wenzel e cols., 2005).

Predominantemente, os dados de sete estudos foram coletados em situação experimental de interação social (conversação) com um auxiliar de pesquisa, com o parceiro de relacionamento afetivo ou com outros participantes (indivíduos ansiosos socialmente e não-ansiosos socialmente). Todos os auxiliares de pesquisa foram previamente treinados para as tarefas de interação social. Apenas no estudo de Thompson e Rapee (2002), os auxiliares de pesquisa foram vistos como outros participantes, ao passo que nos demais foram identificados como tal. As interações dos participantes com o seu interlocutor variaram de 5 a 30 minutos, sendo que a maioria dos estudos adotou uma duração inferior a 10 minutos.

Os auxiliares de pesquisa de quatro estudos foram instruídos para iniciar a conversa ou dar dicas verbais para a sua continuidade toda vez que ocorresse um período de silêncio.

As medidas empregadas nos procedimentos destes estudos foram: (a) subjetivas; (b) objetivas; e (c) fisiológicas. As medidas objetivas se constituíram de filmagens das interações; as subjetivas de aplicação de questionários, escalas ou inventários; e as fisiológicas do exame de condutância da pele, nível de coloração da bochecha ou freqüência cardíaca. A Tabela 2 apresenta os momentos em que tais medidas foram tomadas no decorrer das sessões experimentais.

Tabela 2: Registro das medidas realizadas durante as sessões experimentais dos estudos

\begin{tabular}{|c|c|c|c|}
\hline \multirow{2}{*}{ Estudos } & \multicolumn{3}{|c|}{ Medidas } \\
\hline & Antes & Durante & Depois \\
\hline Bögels e cols. (2002) & $\begin{array}{l}\text { - condutância da pele; } \\
\text { - coloração da bochecha; } \\
\text { - Visual Analogue Scales (VAS). }\end{array}$ & $\begin{array}{l}\text { - condutância da pele; } \\
\text { - coloração da bochecha. }\end{array}$ & $\begin{array}{l}\text { - condutância da pele; } \\
\text { - coloração da bochecha; } \\
\text { - VAS; } \\
\text { - atribuições causais de sucesso e fracasso; } \\
\text { - Escala de avaliação de habilidades sociais. }\end{array}$ \\
\hline Sheffer e cols. (2001) & $\begin{array}{l}\text { - Subjective Units of Distress (SUDS); } \\
\text { - freqüência cardíaca. }\end{array}$ & - freqüência cardíaca. & $\begin{array}{l}\text { - SUDS; } \\
\text { - freqüência cardíaca; } \\
\text { - Escala de Impressão. }\end{array}$ \\
\hline Wenzel e cols. (2005) & $\begin{array}{l}\text { - Fear of Negative Evaluation Scale } \\
\text { (FNE); } \\
\text { - Social Avoidance and Distress Scale } \\
\text { (SAD); } \\
\text { - Dyadic Adjustment Scale (DAS); } \\
\text { - Couples's Problem Inventory (CPI). }\end{array}$ & - filmagem das interações. & - \\
\hline $\begin{array}{l}\text { Baker e Edelmann } \\
\text { (2002) }\end{array}$ & - & - filmagem das interações. & $\begin{array}{l}\text { - escalas de avaliação relacionadas à sen- } \\
\text { sação corporal percebida }\end{array}$ \\
\hline Alden e Mellings (2004) & - & - & $\begin{array}{l}\text { - Questionário de Julgamento Social; } \\
\text { - Focus of Attention Questionnaire (FAQ) }\end{array}$ \\
\hline $\begin{array}{l}\text { Christensen e cols. } \\
\text { (2003) }\end{array}$ & - & - & - Avaliação de traços. \\
\hline $\begin{array}{l}\text { Thompson e Rapee } \\
\text { (2002) }\end{array}$ & - & - filmagem das interações. & $\begin{array}{l}\text { - níveis de ansiedade durante as situações } \\
\text { estruturadas e não-estruturadas. }\end{array}$ \\
\hline
\end{tabular}


Para uma melhor compreensão dos delineamentos de pesquisa empregados, em função das especificidades dos mesmos, os procedimentos de coleta de dados serão detalhados.

Em quatro destes estudos, os participantes receberam a instrução geral para atuar como se estivessem sendo apresentados e conhecendo a outra pessoa com a qual estariam interagindo (Alden \& Mellings, 2004; Baker \& Edelmann, 2002; Christensen e cols., 2003; Thompson \& Rapee, 2002). Os participantes de outros dois estudos receberam a instrução específica para iniciar e manter conversa com os auxiliares de pesquisa (Sheffer e cols., 2001; Bögels e cols., 2002).

No procedimento de Bögels e cols. (2002), os participantes interagiram com dois auxiliares de pesquisa, um do sexo masculino e o outro do sexo feminino, e foram informados de que estes auxiliares julgariam suas habilidades de iniciar e manter conversação, e que esta seria filmada para avaliar seu comportamento. Durante a interação, os auxiliares de pesquisa deixaram visíveis três espelhos grandes, nos quais a metade dos participantes via sua imagem refletida durante a conversa, favorecendo a condição de autoconsciência.

Em Wenzel e cols. (2005), os casais foram instruídos a conversarem acerca de três assuntos: neutro (um evento do dia), negativo (problema) e positivo, com uma duração de dez minutos para cada um destes tópicos.

Após cada interação alternada com outros três indivíduos, os participantes do estudo de Christensen e cols. (2003) foram solicitados avaliar a si próprios (autopercepções), seus parceiros de interação e suas meta-percepções (como eles pensaram que foram vistos pelos parceiros), com base em uma série ampla de características de personalidade, como por exemplo, sociável, simpático, nervoso, calado, em escalas de classificação de 9 pontos, ancoradas entre 1 ("nada") e 9 ("muito").

Em Thompson e Rapee (2002), participante e auxiliar de pesquisa foram deixados pelo experimentador na sala experimental com a desculpa de que iria buscar uma fita de vídeo para gravar a interação de desempenho de papéis. Um gravador de vídeo estava visível ao participante, indicando que estava em funcionamento, embora não contivesse fita alguma. A interação resultante foi considerada como tarefa nãoestruturada. Depois que o experimentador retornou, instruiu o participante e auxiliar de pesquisa para imaginar que estavam em uma festa e tinham que conhecer um ao outro tão bem quanto possível, e informou que seriam filmados. Esta interação compreendeu a tarefa estruturada do estudo. Os dois tipos de tarefas foram objeto de comparação.

No estudo de Alden e Mellings (2004), a pesquisadora inicia a conversa e depois observa, atrás de um espelho unidirecional, a interação do participante com uma auxiliar de pesquisa, tendo como instrução que deveriam se conhecer como se tivessem acabado de ser apresentados. Depois, o participante e a auxiliar de pesquisa preencheram os questionários pós-interação.

Em Sheffer e cols. (2001), os participantes tomaram parte em duas provas de conversação em roleplays, correspondendo às condições com demandas altas e baixas para produzir uma impressão positiva de si. Cada prova foi realizada com uma auxiliar de pesquisa diferente. Na condição com demandas baixas, foi dito aos participantes que a auxiliar de pesquisa seria o foco da avaliação, que ela tinha sido instruída para dar a melhor impressão possível, e que eles deveriam avaliá-la usando a Escala de Impressão. Na condição com demandas altas, foi dito aos participantes que eles eram o foco da avaliação, que deveriam dar a melhor impressão possível à auxiliar de pesquisa, e que ela e um outro assistente de pesquisa os avaliariam usando a Escala de Impressão.

Os procedimentos de coleta de dados dos outros três estudos restantes contaram com medidas objetivas (Horley e cols., 2003), subjetivas (Stopa \& Clark, 2000), e objetivas e subjetivas (Strahan, 2003).

Em Horley e cols. (2003), a medida objetiva se constituiu do monitoramento da exploração visual de três fotos apresentando diferentes expressões faciais aos participantes, por meio de um sistema computadorizado. As faces neutra, alegre e triste foram apresentadas por $10 \mathrm{~s}$ aos participantes, com um intervalo de $15 \mathrm{~s}$ entre os estímulos. Eles foram instruídos para se fixarem no centro da tela até a foto aparecer e, então, olhar para ela de qualquer maneira.

Na pesquisa de Stopa e Clark (2000), foi enviado aos participantes um pacote contendo os questionários e escalas, e solicitado a eles para completá-los de forma independente.

Em Strahan (2003), os participantes universitários preencheram inventários e questionários, abrangendo avaliações de ansiedade, habilidades sociais, ajustamento social e acadêmico, e teste de realização educacional, em pequenos grupos (com 4-5 alunos por sessão) realizadas nas 4-6 primeiras semanas de aula. As informações relacionadas ao desempenho acadêmico dos alunos foram fornecidas, semestralmente, pela secretaria da universidade durante dois anos. 
Com relação à codificação e análise dos dados, os observadores de quatro estudos foram treinados para a avaliação ou dos desempenhos comportamentais dos participantes (Bögels e cols., 2002; Sheffer e cols., 2001; Thompson \& Rapee, 2002;) ou de testes e inventários preenchidos por eles (Wenzel e cols., 2005). Em Stopa e Clark (2000), um segundo avaliador independente classificou os dados dos questionários utilizados para verificar a confiabilidade da categorização do primeiro avaliador. A análise dos dados coletados pelos estudos foi realizada, predominantemente, com base em estatística inferencial.

Quanto aos resultados dos estudos incluídos nesta categoria, observou-se uma concordância entre cinco estudos indicando que indivíduos com fobia social e com níveis altos de ansiedade social apresentaram, em comparação com controles não-clínicos, um repertório de habilidades sociais inferior (Baker \& Edelmann, 2002; Horley e cols., 2003; Strahan, 2003; Wenzel e cols., 2005; Thompson \& Rapee, 2002), além de demonstrarem uma competência social inferior (Baker \& Edelmann, 2002; Strahan, 2003; Wenzel e cols., 2005).

No estudo de Horley e cols. (2003), os sujeitos com fobia social apresentaram evitação de características mais importantes da face, particularmente dos olhos, comprometimento este que pode ser atribuído à atenção mais autofocada e autopercepção negativa apresentadas pelos indivíduos com este transtorno. Os resultados de exploração visual deste estudo ofereceram confirmação empírica para a observação clínica de que indivíduos com fobia social tendem a evitar o contato visual durante interação social.

No estudo de Strahan (2003), a ansiedade social não alcançou significância como um preditor de desempenho acadêmico e permanência na universidade. Entretanto, como reconhece a autora, é possível que níveis altos de ansiedade social exerçam seus efeitos sobre a permanência e o desempenho acadêmico indiretamente, considerando que sua correlação negativa com ajustamento universitário foi moderadamente significativa, e ajustamento universitário teve um efeito claro sobre os índices de sucesso acadêmico.

Três estudos verificaram a influência da estrutura e das demandas da situação sobre a ocorrência de desempenhos sociais dos indivíduos com fobia social, ansiosos socialmente ou não-clínicos. Os resultados de Thompson e Rapee (2002) indicaram que a estrutura das situações de interação social regula diferenças entre o desempenho social de indivíduos ansiosos socialmente e de não-ansiosos, sendo que os indivíduos apresentaram um desempenho social melhor em situa- ções estruturadas comparativamente a situações nãoestruturadas. De acordo com esta conclusão, os resultados do experimento de Sheffer e cols. (2001) revelaram que as demandas altas e baixas para produzir uma impressão positiva de si, inerentes às situações sociais, constituem um fator mediador importante nas relações entre ansiedade, freqüência cardíaca e competência social em sujeitos não-clínicos. Na condição com demandas baixas para produzir uma impressão positiva de si, a competência social maior foi consistentemente associada com menor frequiência cardíaca e menor ansiedade auto-relatada. Na condição com demandas altas para produzir uma impressão positiva de si, competência social maior foi associada apenas com maior freqüência cardíaca.

O estudo de Stopa e Clark (2000), indicou que pacientes com fobia social tendem a interpretar situações sociais ambíguas (p. ex., "você tem visitas para uma refeição e elas saem mais cedo do que você esperava") de maneira negativa e apresentam uma tendência específica para interpretar os eventos sociais moderadamente negativos (p. ex., "você está falando com alguém durante algum tempo e ficou claro que ele não está interessado naquilo que você está dizendo") de maneira mais catastrófica, em comparação com os grupos controle.

Outros três estudos tiveram como foco cotejar a auto-avaliação dos participantes com uma avaliação externa. Dois estudos encontraram concordância quanto às autopercepções negativas de indivíduos com níveis altos de ansiedade social. Em Christensen e cols. (2003), os participantes com níveis altos de ansiedade social viram a si mesmos como menos sociáveis, menos simpáticos, mais nervosos, menos inteligentes e mais distantes na interação com outros participantes. Além disso, foram vistos como menos sociáveis, menos relaxados, mais calados, mais nervosos, mais distantes, e com uma tendência ligeiramente significativa para serem vistos como mais superficiais pelos seus parceiros de interação. Os resultados mostraram que outras pessoas são capazes de detectar algum desconforto em indivíduos ansiosos socialmente nas situações sociais, mas elas não os julgam inferiores em conseqüência disto. Este tipo de informação pode ser usado por diferentes abordagens de tratamento para contrapor algumas das crenças negativas, mantidas pelos indivíduos com fobia social, acerca de como eles são percebidos pelos outros, e as consequiências imaginadas disto.

No estudo de Bögels e cols. (2002), os participantes com ansiedade social alta avaliaram suas habilidades sociais, apresentadas durante a conversa, como 
mais deficitárias do que aqueles com ansiedade social baixa. Entretanto, as diferenças entre as pessoas com ansiedade social alta e baixa com respeito às habilidades auto-avaliadas e avaliadas por auxiliares de pesquisa foram devido a diferenças na demonstração de sintomas de ansiedade (p. ex., movimentos irrequietos), mas não diferenças em comportamento socialmente habilidoso.

Em Alden e Mellings (2004), os participantes com fobia social generalizada foram avaliados por eles próprios e pelas auxiliares de pesquisa como menos habilidosos e sentindo ou parecendo mais ansiosos do que os participantes do grupo controle.

Analisando os resultados obtidos pelos estudos, notou-se que níveis altos de ansiedade social afetaram negativamente o desempenho social, as habilidades de comunicação, as percepções interpessoais e a competência social dos indivíduos com fobia social ou ansiosos socialmente. Os resultados do estudo que visou verificar a associação de ansiedade social e desempenho acadêmico foram inconclusivos a este respeito, representando a única restrição à confirmação da hipótese inicial.

Uma breve discussão será feita acerca destes resultados. Embora possa ser assumido que o elemento avaliado do repertório comportamental de um indivíduo com fobia social ou com níveis altos de ansiedade social, em uma situação planejada, seja em geral representativo do seu comportamento, pode-se questionar se isto realmente representa o seu repertório comportamental. Tal questão aponta para a necessidade de estudos respaldados por uma validade ecológica (grau em que as condições de um estudo são verdadeiras ou aplicáveis para a vida real) e com possibilidade de generalização dos resultados, o que não pôde ser contemplado por nenhum dos estudos incluídos nesta categoria. Além disso, as características de gênero das amostras foram muito circunscritas, sendo constituídas predominantemente por participantes do sexo feminino. Outra limitação potencial do rol de estudos analisados foi a imprecisão dos critérios de confirmação diagnóstica para as amostras de casos e comparação.

Na condução de vários destes estudos é quase inevitável assumir que o tamanho das amostras foi influenciado pela natureza da população estudada. Embora se assuma esta limitação, teria sido desejável contar com uma amostra maior de indivíduos com diagnóstico clinicamente confirmado de fobia social.

Constatou-se a inexistência de um teste, inventário ou escala de avaliação de habilidades sociais padronizado que confira maior validade e confiabilidade aos resultados dos estudos incluídos, visto que utilizaram instrumentos diferentes para esta finalidade.

Conclui-se que o perfil de indivíduos com fobia social generalizada ou níveis altos de ansiedade social caracteriza-se pela presença de prejuízos em suas habilidades sociais.

\section{Modalidades terapêuticas - Aplicação e comparação de intervenções clínicas}

Os estudos incluídos nesta categoria utilizaram delineamentos quase-experimentais e tiveram por objetivo avaliar, direta ou indiretamente, a eficácia do Treinamento em Habilidades Sociais (THS) enquanto abordagem terapêutica para tratamento da fobia social.

A eficácia da aplicação do THS foi testada de forma diversa nos diferentes estudos. Em três desses, a eficácia de tratamento foi comparada com a de outras modalidades terapêuticas, tais como terapia cognitivocomportamental (Van Dam-Baggen \& Kraaimaat, 2000a), terapia de suporte (Cottraux e cols., 2000) e terapia comportamental (Stravynski e cols., 2000), que foram empregadas como controles metodológicos. No estudo de Cottraux e cols. (2000), o THS constituiu um módulo componente da terapia cognitivocomportamental.

Em outro estudo, Van Dam-Baggen e Kraaimaat (2000b) investigaram a eficácia do THS em grupo para pacientes com fobia social generalizada agrupados em subtipos "reticente" e "não-reticente". Os autores definiram como "reticentes" os participantes que apresentaram frequiência baixa de comportamentos sociais, e como "não-reticentes" aqueles que registraram freqüência alta de comportamentos sociais.

Com base em um estudo de caso, Espada, Quiles e Méndez (2002) apresentaram uma intervenção multicomponente, na qual o THS constituiu um módulo de tratamento junto a outras técnicas cognitivo-comportamentais (reestruturação cognitiva, auto-instruções, exposição encoberta e ao vivo, focalização da atenção, respiração profunda e relaxamento).

E ainda em um outro estudo, Bishop (2003) testou a aplicabilidade do sistema de aprendizagem à distância para THS, via internet, para ajudar os participantes a enfrentarem melhor suas dificuldades sociais, convertendo frases e sentenças que eles julgavam confusas ou ofensivas em definições mais concisas e compreensíveis.

Nos estudos de comparação (Van Dam-Baggen \& Kraaimaat, 2000a; Cottraux e cols., 2000; Stravynski e cols., 2000), os autores formularam como hipótese a 
afirmativa da melhor eficácia do THS sobre outras abordagens terapêuticas (terapia cognitvo-comportamental, de suporte e comportamental).

Além do estudo de caso, as amostras dos estudos variaram de 13 a 60 pacientes, adultos, de ambos os sexos, com idade variando entre 18 a 57 anos, que receberam diagnóstico de fobia social, primário ou secundário, segundo os critérios do DSM-IV, com e sem comorbidades. Os participantes foram recrutados, na sua maioria, no ambiente clínico. No estudo de Stravynski e cols. (2000), a amostra procedeu predominantemente da comunidade, seguindo descrições do programa de tratamento em vários meios de comunicação. Apenas o estudo de Bishop (2003) não relatou a procedência da amostra. Quatro estudos (Van DamBaggen \& Kraaimaat, 2000a; Van Dam-Baggen \& Kraaimaat, 2000b; Cottraux e cols., 2000; Stravynski e cols., 2000) apresentaram como critérios comuns de exclusão, a presença de indicadores de transtornos psicóticos e adição a substâncias.

Ainda no que diz respeito aos critérios de inclusão de participantes, observou-se uma diversidade de condições destacadas nos diferentes estudos. Van DamBaggen e Kraaimaat (2000a) utilizaram um procedimento de emparelhamento dos participantes para as duas condições de tratamento, visando garantir duas amostras razoavelmente equivalentes. Em outro estu- do (Van Dam-Baggen \& Kraaimaat, 2000b) que incluiu pacientes internos de uma clínica psiquiátrica, a designação para a composição da amostra "reticente" e "não-reticente" teve por base os escores na Frequency Scale do Inventory of Interpersonal Situations (IIS). Nos estudos de Cottraux e cols. (2000) e Stravynski e cols. (2000), os participantes foram aleatoriamente distribuídos para os grupos de tratamento. No caso clínico apresentado por Espada e cols. (2002), o participante era um paciente identificado que buscou espontaneamente o atendimento. Bishop (2003) não fez menção alguma acerca do procedimento de seleção dos participantes para o estudo. Os pacientes identificados em três estudos (Cottraux e cols., 2000; Espada e cols., 2002; Stravynski e cols., 2000) tiveram em comum o fato de buscarem espontaneamente atendimento, e apenas em dois estudos a alocação nos grupos foi aleatória.

Cada um dos estudos empregou um conjunto de instrumentos e medidas diferentes dos demais. O Symptom Checklist-90 (SCL-90) foi o único que se repetiu em três estudos (Van Dam-Baggen \& Kraaimaat, 2000a; 2000b; Stravynski e cols., 2000).

A Tabela 3 apresenta uma caracterização detalhada dos delineamentos adotados nos estudos sobre a eficácia do THS.

Tabela 3. Caracterização dos delineamentos adotados nos estudos de eficácia

\begin{tabular}{|c|c|c|c|c|c|}
\hline \multirow[b]{2}{*}{ Delineamentos } & \multicolumn{5}{|c|}{ Estudos } \\
\hline & $\begin{array}{l}\text { v. Dam-Baggen e } \\
\text { Kraaimaat (2000a) }\end{array}$ & $\begin{array}{c}\text { v. Dam-Baggen e } \\
\text { Kraaimaat (2000b) }\end{array}$ & $\begin{array}{l}\text { Cottraux e } \\
\text { cols. }(2000)\end{array}$ & $\begin{array}{c}\text { Stravynski } \\
\text { e cols. }(2000)\end{array}$ & $\begin{array}{l}\text { Espada e } \\
\text { cols. (2002) }\end{array}$ \\
\hline Aplicação: - grupo & + & + & + & + & - \\
\hline - individual & - & - & - & - & + \\
\hline Duração das sessões: & $90 \mathrm{~min}$ & $\mathrm{sr}$ & $120 \mathrm{~min}$ & $120 \mathrm{~min}$ & $50 \mathrm{~min}$ \\
\hline № de participantes: & 5 a 8 & 8 a 10 & 4 a 6 & sr & 1 \\
\hline Terapeuta: - único & + & + & - & + & + \\
\hline - dupla & - & - & + & - & - \\
\hline - co-terapeuta & + & - & - & + & - \\
\hline Técnicas: - ensaio comp. & + & + & + & + & + \\
\hline - modelagem & + & + & + & + & + \\
\hline - tarefas de casa & + & + & + & + & + \\
\hline - feedback & - & - & + & + & + \\
\hline - reforçamento & - & - & + & + & + \\
\hline - biblioterapia & + & + & + & - & - \\
\hline - apr. sucessiva & + & + & - & - & - \\
\hline - an. funcional & - & - & - & + & + \\
\hline - instruções & - & - & - & + & + \\
\hline - automonit. & + & + & - & + & + \\
\hline - modelação & - & - & - & - & + \\
\hline - fase educativa & - & - & - & - & + \\
\hline - estr. sol. prob. & + & + & - & - & - \\
\hline № de sessões: & 20 & 20 & 6 & 14 & 4 \\
\hline Seguimento: & $3 m$ & - & 6 e $12 \mathrm{~m}$ & 6 e $12 \mathrm{~m}$ & $12 \mathrm{~m}$ \\
\hline
\end{tabular}

comp.: comportamental; apr.: aproximação; an.: análise; automonit.: automonitoramento; estr. sol. prob.: estratégias de solução de problemas; m: meses; (+): presente nos estudo; (-): ausente no estudo; sr: sem referência. 
Como pode ser observado, o THS foi administrado em grupo em quatro estudos. Na intervenção multicomponente de Espada e cols. (2002) e no sistema de aprendizagem à distância de Bishop (2003), o THS foi aplicado individualmente. A predominância de aplicação do THS no formato grupal, em detrimento ao individual, pode ser justificada por apresentar as seguintes vantagens: (a) representa economia de tempo para o terapeuta; (b) oferece maior diversidade de ensaio comportamental com um número maior de pessoas; (c) possibilita generalização mais rápida dos ganhos e uma maior quantidade de feedback efetivo para os desempenhos treinados; (d) proporciona experiência com um número maior de situações-problema e mais suporte para solucioná-las; (e) possui maior disponibilidade de modelos múltiplos, além dos fornecidos pelo terapeuta; e (f) favorece aprendizagem de discriminação das possíveis conseqüências desejáveis e indesejáveis para emissão dos novos comportamentos aprendidos.

Os grupos de THS descritos foram constituídos de quatro a dez participantes e em um deles não foi mencionado o número de participantes que compuseram os grupos de tratamento. Quanto à duração das sessões, estas variaram de 90 a 120 minutos. O número total de sessões variou de seis a vinte sessões. No estudo de caso, o módulo de THS incluído no tratamento multicomponente durou quatro sessões de cinqüenta minutos.

As intervenções clínicas em Van Dam-Baggen e Kraaimaat (2000a; 2000b), Cotraux e cols. (2000) e Stravynski e cols. (2000) foram realizadas por terapeutas experientes em THS ou terapia cognitivocomportamental, contando com um co-terapeuta em duas dessas intervenções.

Tendo como base os estudos de Van Dam-Baggen e Kraaimaat (2000a; 2000b) e Espada e cols. (2002), as seguintes habilidades sociais foram incluídas no programa de THS para pacientes com fobia social generalizada: observar; ouvir; iniciar, manter e encerrar conversação; dar e receber feedback; contato visual; modulação do volume e entonação da fala; fazer e recusar pedidos; aceitar recusas; fazer queixas; cumprimentar; declarar auto-afirmações positivas; fazer e receber críticas; expressar opiniões; e defender os próprios direitos. No estudo de Espada e cols. (2002), a fase educativa compreendeu ensinar o paciente sobre os estilos de desempenho social passivo, assertivo e agressivo; sobre os componentes do desempenho social; e a definição de comportamento socialmente habilidoso. Observou-se que a assertividade esteve presente de forma explícita em quatro intervenções clínicas do THS (Cottraux e cols., 2000; Espada e cols., 2002; Van Dam-Baggen \& Kraaimaat, 2000a; 2000b).

As técnicas típicas empregadas em programas de THS e comuns a todos os estudos de intervenção clínica foram: ensaio comportamental, modelagem e atribuição de tarefas de casa. O emprego de biblioterapia foi comum a três estudos, e as técnicas de feedback e reforço positivo estiveram presentes também em três estudos. O procedimento de análise funcional foi empregado em apenas dois estudos.

A atribuição de tarefas de casa tem sido um dos recursos utilizados para verificar a generalização dos efeitos de programas de THS (Del Prette \& Del Prette, 2005). Além disso, as tarefas de casa também permitem aos participantes a discriminação de características da situação natural diante das quais o desempenho das habilidades recém-adquiridas pode gerar conseqüências desejáveis ou indesejáveis (discriminação de consequiências), visando assim emissão de outros comportamentos alternativos. Em termos analíticoconceituais, a atribuição de tarefas de casa serve como uma ferramenta para a identificação de problemas de controle de estímulos que estejam dificultando a eficácia da intervenção sobre o funcionamento social do indivíduo em seu ambiente natural. Por conseguinte, esta eficácia encontra-se implicada na validade social das aquisições previstas pelos programas de THS.

Em todas as intervenções do THS aplicadas em contextos clínicos, foram realizadas avaliações antes e depois do tratamento. Avaliações de segmento foram aplicadas em quatro estudos, havendo uma variabilidade de período entre eles, como demonstrado pela Tabela 3.

A maioria dos estudos incluídos nesta categoria utilizou a estatística inferencial para o tratamento e análise dos dados coletados, com exceção do estudo de Espada e cols. (2002) que comparou os dados de auto-registro (freqüência do comportamento-problema e intensidade do desconforto) e os escores obtidos nas medidas empregadas, e de Bishop (2003) que comparou as médias das atitudes favoráveis dos participantes, pontuadas em uma escala de gradação, em relação ao sistema de aprendizagem à distância.

Uma desvantagem do fato da maioria dos participantes dos estudos terem sido recrutados no ambiente clínico é a possibilidade deste contexto representar os casos mais graves de fobia social, o que, em última 
análise, teria implicações para a generalização dos dados. Referendando esta limitação, observou-se que os participantes recrutados neste contexto receberam, predominantemente, o diagnóstico primário ou secundário de fobia social generalizada, com e sem comorbidades.

Com relação aos resultados obtidos, a eficácia do THS foi comprovada pela maioria dos estudos, inclusive em comparação às outras modalidades de tratamento, tais como a terapia cognitivo-comportamental e a terapia de suporte, confirmando a hipótese inicial. No estudo de Stravynski e cols. (2000), embora o THS tenha produzido melhores resultados mais rapidamente, esta tendência se nivelou com a terapia comportamental, e ambos os tratamentos resultaram em um nível igual de melhora na avaliação de segmento após doze meses. Na intervenção multicomponente de Espada e cols. (2002), o paciente apresentou melhora notável em suas relações sociais, especialmente frente às conversações e aos eventos sociais. No estudo de Bishop (2003), os participantes com fobia social generalizada indicaram se perceberem mais capazes de compreender as histórias, mas consideraram que o sistema de aprendizagem à distância não fez com que se sentissem melhores, além de demonstrarem uma atitude um pouco negativa com relação à funcionalidade do mesmo.

A validade interna do THS poderia se beneficiar muito se ele fosse contrastado com uma condição controle ("placebo") convincente, ou seja, uma condição planejada para se assemelhar a uma terapia sem sê-lo. Esta proposição ganha consistência considerando que, provavelmente, um período na lista de espera não seria o melhor controle para os efeitos de tratamento, além da falta de uma definição precisa do que constituiria a melhor condição de controle para intervenções psicossociais.

Em cinco estudos, a eficácia tanto do THS quanto das outras modalidades terapêuticas foi comprovada pelos dados de medida de auto-relato. Apenas os estudos de Stravynski e cols. (2000) e de Espada e cols. (2002) utilizaram entrevistas clínicas para avaliar a eficácia dos tratamentos oferecidos, além das medidas de auto-relato.

Entretanto, uma ressalva precisa ser feita com relação à eficácia atestada por esses estudos. Analisandose as metodologias empregadas, apenas os estudos de Cottraux e cols. (2000) e Stravynski e cols. (2000) podem ser caracterizados como estudos de eficácia, que supõem a existência de randomização para os grupos de intervenção, na composição de seus delineamentos de pesquisa. Os demais estudos (Van DamBaggen \& Kraaimaat, 2000a; 2000b; Espada e cols., 2002) se propuseram a testar eficácia quando não o fizeram, sendo melhor caracterizados como estudos de efetividade, que não requerem alocação randômica de participantes nos grupos.

Algumas considerações precisam ser feitas com relação aos resultados de três estudos realizados em contextos clínicos. No estudo de Cottraux e cols. (2000), qualquer conclusão sobre a eficácia relativa às fases de terapia cognitiva, que ocorreu primeiro, e THS seria incerta, embora a maior parte da mudança foi vista depois do módulo de THS. Supostamente, a terapia cognitiva abriu caminho para o THS, uma vez que os pacientes puderam continuar usando técnicas cognitivas durante o restante da intervenção. Neste sentido, pode-se considerar que uma ação sinérgica da terapia cognitiva mais THS pode ter influenciado os dados. Isto também pode ter estado presente no estudo de caso de Espada e cols. (2002), visto que várias técnicas cognitivas e comportamentais antecederam o módulo de THS. Mesmo não se tratando de estudos clínicos cruzados (cross-over), uma aplicação alternada entre os procedimentos em amostras diferentes seria desejável para garantir melhor controle metodológico aos delineamentos propostos, o que também não evitaria o problema dos efeitos da primeira intervenção manterem-se durante o período em que a segunda fosse dada.

Em Stravynski e cols. (2000), a terapia comportamental foi planejada para adotar a forma do THS, visando melhorar como o paciente desempenhava espontaneamente o comportamento-alvo escolhido, com a única restrição de não utilizar determinadas técnicas típicas de programas de treinamento, como a modelação, ensaio comportamental e feedback. Pela descrição oferecida pelos autores, houve uma convergência entre a terapia comportamental e o THS nos seguintes pontos: teve como base um foco interpessoal forte e visou ensinar o paciente tanto a buscar criar circunstâncias sociais novas como a se engajar em todas situações sociais de uma maneira diferente. Estas proposições em comum sugerem que a terapia comportamental proposta pelos autores se apresentou como uma interface do THS, sem a aplicação de todas as técnicas utilizadas em programas típicos de treinamento.

A funcionalidade do sistema de aprendizagem à distância proposto por Bishop (2003) apresenta algumas limitações e implicações para sua utilização em 
situações sociais reais. Em primeiro lugar, o sistema teria que ser adaptado para satisfazer as necessidades dos indivíduos com fobia social generalizada, uma vez que os mesmos não têm problemas para interpretar o significado literal do que lhes é dito em situações sociais, mas sim por interpretarem comentários de maneira negativa. Em segundo lugar, a aplicação prática deste sistema exigiria um processamento cognitivo adicional de informações dos indivíduos para participarem em uma situação social, além de fazê-los perderem contato visual com o interlocutor, o que amplificaria os seus sintomas de prejuízo social.

Devido aos resultados dos estudos atestando a sua aplicabilidade no contexto clínico, o THS pode ser um dos melhores tratamentos de escolha para pacientes psiquiátricos com diagnóstico primário ou secundário de fobia social generalizada.

Tendo em vista as descrições e os resultados dos estudos, pode-se concluir que o melhor delineamento para testar a eficácia do THS em pacientes com diagnóstico de fobia social seria aquele no qual esta modalidade terapêutica constituísse abordagem única de tratamento, sendo administrada no formato grupal, com alocação randômica dos participantes nos grupos de treinamento e de comparação, e utilizando-se de tarefas de interação contextualizadas com base em avaliação prévia dos recursos e déficits das habilidades apresentadas pelos participantes em diferentes contextos. Parece que os delineamentos de THS com melhores resultados foram os utilizados nos estudos de Van Dam-Baggen e Kraaimaat (2000a; 2000b), embora não possam ser caracterizados de forma estrita como estudos de eficácia.

No que diz respeito aos resultados de seu estudo, Stravynski e cols. (2000) reconheceram numerosas divergências entre os escores obtidos na Social Adjustment Scale (SAS) e os registros das entrevistas clínicas, levantando questões óbvias de validade. Analisando os resultados que atestaram a "eficácia" do THS entre os estudos, pode-se concluir que o alcance desta modalidade terapêutica teve como fator limitador o caráter inferencial das escalas na avaliação da aquisição de habilidades sociais e outros ganhos terapêuticos pelos pacientes, deixando questões de validade ecológica em aberto.

\section{COMENTÁRIOS FINAIS}

Examinando os 16 estudos agrupados nas duas categorias propostas, constatou-se a inexistência de um instrumento ou medida padrão para avaliação de ha- bilidades sociais que confira uma maior validade e confiabilidade aos dados coletados, levando em conta a diversidade de instrumentos utilizados com este objetivo. No conjunto de artigos analisados, observouse também a necessidade de reconhecimento de um instrumento sistematicamente estudado como "padrão-ouro" para a avaliação de habilidades sociais e/ou competência social dos indivíduos.

Verificou-se que os estudos sobre a eficácia do THS não mantiveram uma homogeneidade de procedimentos adotados quanto ao número de sessões e técnicas empregadas nas intervenções, não oferecendo, portanto, um modelo de delineamento típico que possa ser prescrito para o tratamento de fobia social.

Considera-se que o excesso de ansiedade interpessoal e as dificuldades de processamento em termos cognitivo-afetivos (vieses interpretativos, auto e metapercepções negativas, e atenção autofocada) dos indivíduos com fobia social possam estar implicados nos déficits de desempenho ou de fluência apresentados por eles, inibindo assim a exibição de um desempenho socialmente competente em suas interações sociais, caso não atestem um déficit de aquisição.

No artigo de Thompson e Rapee (2002), parece que o termo "treinamento em habilidades sociais" está sendo usado como se fosse sinônimo de instalar habilidades sociais nas pessoas. Neste ponto, aparentemente, os autores não consideram que o THS não visa somente instalar novas habilidades sociais no repertório comportamental dos indivíduos que apresentam déficits nesse repertório, mas também melhorar o desempenho social deles diante de demandas específicas das situações interpessoais, assim como promover a competência social frente a audiências distintas. Além disso, o THS também abrange a redução de ansiedade enquanto objetivo alcançável, não apenas para indivíduos fóbicos sociais, por meio de técnicas apropriadas e específicas para tal.

Os resultados do estudo de Christensen e cols. (2003) evidenciaram que as meta-percepções negativas de indivíduos ansiosos socialmente eram mais uma função de suas próprias autopercepções negativas do que as percepções negativas dos outros, encontrando consonância com a afirmação de Furmark (2000) de que a autopercepção de indivíduos com fobia social gera uma impressão negativa de si mesmo que, para eles, reflete o que os outros realmente notam e pensam sobre eles.

Estando de acordo com os argumentos de Wells e Clark (1997), os resultados dos estudos de Alden e 
Mellings (2004) mostraram que o indivíduo socialmente fóbico tipicamente constrói uma imagem negativa de si mesmo a partir da perspectiva de um observador externo.

Estudos futuros com melhor controle metodológico precisam ser realizados para uma verificação mais acurada de possíveis associações entre ansiedade social e desempenho acadêmico.

Concluiu-se que é de suma importância a caracterização prévia do repertório de habilidades sociais dos indivíduos em geral e, particularmente dos fóbicos sociais e ansiosos socialmente, para propor programas de tratamento efetivos que atendam de modo mais direto e objetivo as suas demandas interpessoais.

A análise do alcance dos resultados atestando a eficácia do THS e dos indicadores de habilidades sociais avaliados evidenciou a necessidade de novos estudos com amostras clínicas e não-clínicas, com alocação randômica dos participantes, com diagnóstico preciso e proposição de tarefas de interação contextualizadas, respaldados por uma validade ecológica que contemple a generalização dos resultados obtidos quanto à associação das habilidades sociais e fobia social, podendo assim evidenciar a funcionalidade e o processo pela qual a ansiedade interfere no desempenho social dos indivíduos.

\section{REFERÊNCIAS}

Alden, L. E. \& Mellings, T. M. B. (2004). Generalized social phobia and social judgments: The salience of self- and partnerinformation. Journal of Anxiety Disorders, 18, 143-157.

American Psychiatric Association. (1994). Diagnostic and statistical manual of mental disorders ( $4^{\mathrm{a}}$ ed.). Washington DC.

Angélico, A. P. (2004). Estudo descritivo do repertório de habilidades sociais de adolescentes com síndrome de Down. Dissertação de Mestrado não publicada. Programa de PósGraduação em Educação Especial: Universidade Federal de São Carlos. São Carlos, SP, 126 p.

Argyle, M. (1994). Psicologia del comportamiento interpersonal. Madrid: Alianza Universidad. (Originalmente publicado em 1967)

Baker, S. R. \& Edelmann, R. J. (2002). Is social phobia related to lack of social skills? Duration of skill-related behaviours and ratings of behavioural adequacy. British Journal of Clinical Psychology, 41(3), 243-257.

Bandeira, M. (2003). Avaliando a competência social de pacientes psiquiátricos: Questões conceituais e metodológicas. Em A. Del Prette \& Z. A. P. Del Prette (Orgs.), Habilidades sociais, desenvolvimento e aprendizagem: Questões conceituais, avaliação e intervenção (pp. 207-234). Campinas: Alínea.

Bishop, J. (2003). The internet for educating individuals with social impairments. Journal of Computer Assisted Learning, 19(4), 546-556.
Bögels, S. M., Rijsemus, W. \& De Jong, P. J. (2002). Self-focused attention and social anxiety: The effects of experimentally heightened self-awareness on fear, blushing, cognitions, and social skills. Cognitive Therapy \& Research, 26(4), 461-472.

Christensen, P. N., Stein, M. B. \& Means-Christensen, A. (2003). Social anxiety and interpersonal perception: A social relations model analysis. Behaviour Research and Therapy, 41, 13551371.

Cottraux, J., Note, I., Albuisson, E., Yao, S. N., Note, B., Mollard, E., Bonasse, F., Jalenques, I., Guérin, J. \& Coudert, A. J. (2000). Cognitive behavior therapy versus supportive therapy in social phobia: A randomized controlled trial. Psychotherapy and Psychosomatics, 69(3), 137-146.

Del Prette, A. \& Del Prette, Z. A. P. (2001). Psicologia das relações interpessoais: Vivências para o trabalho em grupo. Rio de Janeiro: Vozes.

Del Prette, A. \& Del Prette, Z. A. P. (2005). A importância das tarefas de casa como procedimento para a generalização e validação do treinamento de habilidades sociais. Em $\mathrm{H}$. Guilhardi \& N. C. de Aguirre (Orgs.), Primeiros passos em análise do comportamento e cognição (pp. 67-74). Santo André: ESETec Editores Associados.

Espada, J. P., Quiles, M. J. \& Mendez, F. X. (2002). Terapia cognitiva y exposición mixta en un caso de fobia social. Análisis y Modificación de Conducta, 28(117), 129-160.

Furmark, T. (2000). Social phobia: From epidemiology to brain function. Acta Universitatis Upsaliensis. Comprehensive Summaries of Uppsala Dissertations from the Faculty of Social Sciences 97. Uppsala. 72 p.

Halford, W. K. \& Hayes, R. L. (1995). Social skills in schizophrenia: Assessing the relationship between social skills, psychopathology and community functioning. Social Psychiatry and Psychiatric Epidemiology, 30(1), 14-19.

Horley, K., Williams, L. M., Gonsalvez, C. \& Gordon, E. (2003). Social phobics do not see eye to eye: A visual scanpath study of emotional expression processing. Journal of Anxiety Disorders, $410,1-12$.

Morrison, R. L. \& Bellack, A. S. (1987). Social functioning of schizophrenic patients: Clinical and research issues. Schizophrenia Bulletin, 13(4), 715-725.

Sheffer, C. E., Penn, D. L. \& Cassisi, J. E. (2001). The effects of impression management demands on heart rate, self-reported social anxiety, and social competence in undergraduate males. Journal of Anxiety Disorders, 15(3), 171-182.

Stopa, L. \& Clark, D. M. (2000). Social phobia and interpretation of social events. Behaviour Research and Therapy, 38, 273-283.

Strahan, E. Y. (2003). The effects of social anxiety and social skills on academic performance. Personality and Individual Differences, 34(2), 347-366.

Stravynski, A., Arbel, N., Bounader, J., Gaudette, G., Lachance, L., Borgeat, F., Fabian, J., Lamontagne, Y., Sidoun, P. \& Todorov, C. (2000). Social phobia treated as a problem in social functioning: A controlled comparison of two behavioural group approaches. Acta Psychiatrica Scandinavica, 102(3),188198.

Thompson, S. \& Rapee, R. M. (2002). The effect of situational structure on the social performance of socially anxious and non-anxious participants. Journal of Behavior Therapy and Experimental Psychiatry, 33(2), 91-102. 
Turner, S. M., Beidel, D. C. \& Townsley, R. M. (1992). Behavioral treatment of social phobia. Em S. M. Turner, K. S. Calhoum \& H. E. Adams (Orgs.), Handbook of clinical behavior therapy ( $2^{\mathrm{a}}$ ed.) (pp. 13-37). Nova Iorque: Willey.

Van Dam Baggen, R. \& Kraaimaat, F. (2000a). Group social skills training or cognitive group therapy as the clinical treatment of choice for generalized social phobia? Journal of Anxiety Disorders, 14(5), 437-51.

Van Dam Baggen, R. \& Kraaimaat, F. W. (2000b). Social skills training in two subtypes of psychiatric inpatients with generalized social phobia. Scandinavian Journal of Behaviour Therapy, 29(1), 14-21.

Wells, A. \& Clark, D. M. (1997). Social phobia: A cognitive approach. Em G. C. L. Davey (Org.), Phobias: A handbook of theory, research and treatment (pp. 3-26). Chichester: John Wiley e Sons.

Wenzel, A., Graff-Dolezal, J., Macho, M. \& Brendle, J. R. (2005). Communication and social skills in socially anxious and nonanxious individuals in the context of romantic relationships. Behaviour Research and Therapy, 43, 505-519.

Zigler, E. \& Phillips, L. (1962). Social competence and the process-reactive distinction in psychopathology. Journal of Abnormal and Social Psychology, 6, 215-223.

Recebido: 20/12/2005

Revisado: 06/06/2006

Aceito: 15/06/2006

\section{Sobre os autores:}

Antonio Paulo Angélico: Doutorando pelo Programa de Pós-Graduação em Saúde Mental da Faculdade de Medicina de Ribeirão Preto da Universidade de São Paulo (FMRP - USP) e Mestre em Educação Especial pelo Programa de Pós-Graduação em Educação Especial da Universidade Federal de São Carlos (UFSCar). Endereço para correspondência: Programa de Pós-Graduação em Saúde Mental, Rua Tenente Catão Roxo, 2650 -14048-900 - Ribeirão Preto/SP - Endereço eletrônico: angelico@ rnp.fmrp.usp.br.

José Alexandre de Souza Crippa: Professor Doutor do Departamento de Neurologia, Psiquiatria e Psicologia Médica da Faculdade de Medicina de Ribeirão Preto da Universidade de São Paulo. Endereço para correspondência: Programa de Pós-Graduação em Saúde Mental, Rua Tenente Catão Roxo, 2650 - 14048-900 - Ribeirão Preto/SP - Endereço eletrônico: jcrippa@ fmrp.usp.br.

Sonia Regina Loureiro: Professora Doutora do Departamento de Neurologia, Psiquiatria e Psicologia Médica da Faculdade de Medicina de Ribeirão Preto da Universidade de São Paulo. Endereço para correspondência: Programa de Pós-Graduação em Saúde Mental, Rua Tenente Catão Roxo, 2650 - 14048-900 - Ribeirão Preto/SP - Endereço eletrônico: srlourei@ fmrp.usp.br 